\title{
Adaptive Service-Chain Routing for Virtual Network Functions in Software-Defined Networks
}

\author{
Abhishek Dwaraki and Tilman Wolf \\ Department of Electrical and Computer Engineering \\ University of Massachusetts, Amherst, MA, USA \\ \{adwaraki,wolf\}@umass.edu
}

\begin{abstract}
Software-defined networking is shifting data communication networks toward more centralized control. The availability of virtual machines and lightweight containers enables dynamic placement of virtual network functions on demand. A key challenge is to efficiently route service-chain requests and place processing functions in a network under operational constraints. We present a novel method of solving the node-constrained service chain routing problem in a software-defined network. The main idea is to transform the network representation to a layered graph that considers processing steps and allows the use of conventional shortestpath algorithms, such as Dijkstra's algorithm, to solve the problem. We demonstrate the feasibility of this approach through an emulated prototype implementation that uses dynamic utilization measurements on links and hosts to determine network conditions at runtime.
\end{abstract}

\section{Categories and Subject Descriptors}

\section{C.2.0 [Computer-Communication Networks]: General-} Data communications

\section{Keywords}

service chaining, network function virtualization, softwaredefined networking, layered graph

\section{INTRODUCTION}

Software-Defined Networking (SDN) is an emerging technology for controlling networks at a finer granularity than is possible in current networks. An SDN controller can set up the path of a flow through its network by configuring each switch along the way to match packets of this flow and

Permission to make digital or hard copies of all or part of this work for personal or classroom use is granted without fee provided that copies are not made or distributed for profit or commercial advantage and that copies bear this notice and the full citation on the first page. Copyrights for components of this work owned by others than ACM must be honored. Abstracting with credit is permitted. To copy otherwise, or republish, to post on servers or to redistribute to lists, requires prior specific permission and/or a fee. Request permissions from permissions@acm.org.

HotMiddlebox, August 22-26, 2016, Florianopolis, Brazil

(c) 2016 ACM. ISBN 978-1-4503-4424-1.

DOI: http://dx.doi.org/10.1145/2940147.2940148 forward them to a specific next hop [2,9]. New flows can be set up dynamically when the first packet of a connection arrives at the edge of the SDN network (and the switch does not find a matching rule). The controller is then informed of the new packet and computes a suitable path for the packet. This allows for great flexibility in terms of network control and management.

An interesting aspect of SDN is the ability to not only consider forwarding of network traffic, but also its processing. Such "network functions" or "network services" [13] can implement header processing and payload processing functions, such as network address translation (NAT), firewalling, or virtual private network (VPN) termination. These functions, called virtual network functions (VNFs) can be implemented in software on conventional processing systems that are co-located with networking equipment. The sequence of functions that need to be set up for a specific flow is referred to as a "service chain."

In traditional IP networks, nodes that implement such functions need to be located in the path of traffic (e.g., a firewall at the ingress point to a network). However, in SDN, where the path of each flow can be determined independently, it is possible to virtualize the implementation of these network functions. Through network function virtualization (NFV), the location of the processing node that implements the function can be anywhere within the SDN network or placed on a pre-computed path from source to destination.

This approach provides significant scalability benefits since multiple virtual nodes can implement processingintense functions, such as VPN termination, for different flows independently in a distributed manner. However, multiple potential processing nodes pose interesting routing challenges. Specifically, these challenges are: (1) determining a flow path that traverses suitable processing nodes in the correct order to meet the requirements of a given service chain, and (2) considering network load and other dynamic characteristics when routing through existing VNFs.

We address these challenges in this paper. We present an algorithm that can be used to compute paths efficiently, meeting all the requirements stated above. The primary idea is to transform the traditional routing problem in networks by merely modifying the structure of the network graph. Using a suitable metric that combines both link costs and pro- 
cessing costs, conventional shortest path algorithms can be used to solve this problem. To reflect dynamics, we exploit the ability of SDN controllers to collect run-time statistics from switches in the network to assess dynamic loads. We use models from queuing theory to translate this information into dynamic cost values for the placement problem.

Specifically, the contributions of our paper are:

- Design of a Adaptive Service Routing (ASR) algorithm that uses a layered graph construction. Dijkstra's shortest path algorithm [5] is then used to determine the best path for a VNF chain.

- Use of cost functions that consider the dynamics of networks, such as load on links and processing nodes, to enable optimal dynamic path routing.

- Evaluation of the ASR algorithm to validate its correctness, both theoretically and on an SDN framework with ONOS [1], Mininet [8] and sFlow [12].

We believe that this work presents an important step toward realizing the full potential of network function virtualization in SDNs, especially in the context of dynamic adaptation under changing network loads.

\section{RELATED WORK}

SDNs switch flows and flow aggregates rather than IP aggregates as in the current Internet [2,9]. Control mechanisms in SDN are centrally located in the SDN controller, rather than distributed across network nodes. The idea of service chains, as they are implemented in NFV today, have been alluded to in our prior work $[6,13]$ and other approaches.

Path-finding in network graphs is an important problem and many efficient path-finding algorithms have been proposed. Our ideas for using a layered graph in Adaptive Service Routing are based on prior work in [4]. The work presented here expands on this by considering the dynamics of SDNs and how to implement such an approach in practice.

Placement of virtual network functions has been explored in [7]. Their approach considers more complex metrics, such as host resource consumption, but represent also a more complex solution. Similarly, multi-criteria path finding techniques consider multiple metrics, e.g., [3]. We, however, focus on a single metric, since this approach leads to efficient and effective solutions to VNF placement.

\section{ORDER-CONSTRAINED NET- WORK FUNCTION ROUTING PROBLEM}

Network functions implement operations on network traffic, specifically on the packets that are transmitted over the network. The operations are dependent on the type of traffic. We assume that this distinction is made at the granularity of flows and flow aggregates, both of which can be represented by match-fields defining the values (or wild-cards) of different header fields. Within a network controlled by a single administrative entity, the problem is to determine a path for a flow that connects its end-points (either end-systems or ingress or egress points to the network) and traverses a set of nodes where network functions exist or can be instantiated.

\subsection{Problem Statement}

The network can be represented by a graph $G=(V, E)$ with $n$ vertices $V=\left\{v_{1}, v_{2}, \ldots v_{n}\right\}$ and $m$ directed edges $E=\left\{\left(e_{i}, e_{j}\right), \ldots\right\}$ connecting these vertices. Each edge $\left(e_{i}, e_{j}\right)$ has an associated cost $c\left(e_{i}, e_{j}\right)$. We define the set of $t$ network functions that are implemented in this network as $F=\left\{f_{1}, f_{2}, \ldots, f_{t}\right\}$. Each vertex may implement some of these network functions, which are specified by the subset of $F$ provided by function $f(v)$. A connection, $c=\left(v_{s}, v_{t},\left(r_{1}, r_{2}, \ldots, r_{k}\right)\right)$, is specified by the starting vertex, $v_{s}$, the terminating vertex, $v_{t}$, and the sequence of $k$ requested network functions, $\left(r_{1}, r_{2}, \ldots, r_{k}\right)$.

The network function placement problem is to determine a mapping, $M=(a(\cdot), p(\cdot, \cdot))$, that consists of an assignment function, $a$, and a path function, $p$. The assignment function, $a\left(r_{i}\right)$, assigns each function, $r_{i}$, of a connection to certain vertices in the graph. The path function, $p\left(a\left(r_{i}\right), a\left(r_{i+1}\right)\right)$, determines the sequence of vertices traversed between functions (and between the starting vertex and the first function and the last function and the terminating vertex). When we say assignment, this does not mean that we instantiate the VNF on a particular node. It means that we have prior knowledge of where the VNFs are located and we map them to their respective nodes.

A path $P=p_{1} \ldots p_{l}$ computed by the path function is considered to be a valid, admissible path that services the virtualized function chain if there exist integers $i_{1} \ldots i_{l}$ such that $1 \leq i_{1} \leq \cdots \leq i_{l} \leq n$ and the function provided at $p_{i_{j}}$, $f\left(p_{i_{j}}\right) \in f(v)$ for $i \leq j \leq l$.

\subsection{Constraints and Optimization Metrics}

We restrict ourselves to a path-finding algorithm that uses a single criterion (multi-criteria path finding approaches are briefly discussed in Section 2). The constraints presented by the VNF routing problem are twofold: (1) find a path from source to destination with least cost, and (2) maintain the order of services requested for provisioning.

A critical aspect of the Adaptive Service Routing algorithm is that the metric captures cost for both communication and processing. There are many potential choices for such a cost (e.g., actual financial cost of use, delay, probability of meeting QoS requirements, etc.) and more complex multicriteria cost functions could be used. For our work, we use delay as the single cost metric for both communication and processing. The use of delay yields practical solutions since it is an important consideration in many networks. Also, delay lends itself well to representing dynamic loads on network links and on VNF processing nodes.

\section{ADAPTIVE SERVICE ROUTING}

This section describes how the network graph can be transformed to help solve the complex service-chain routing problem using conventional methods. 


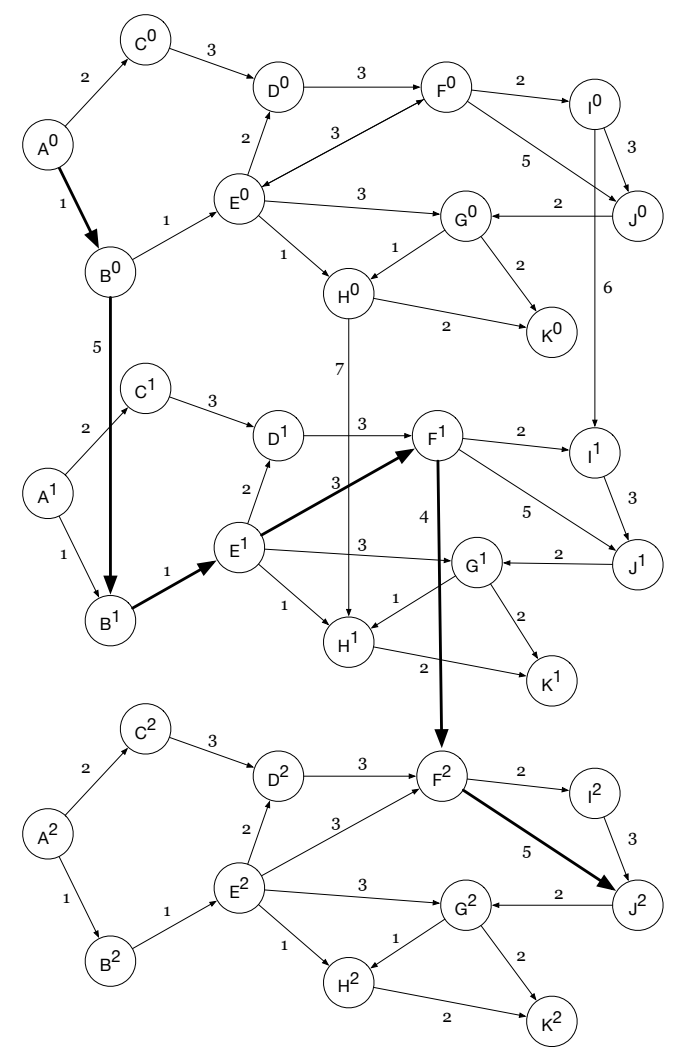

Figure 1: VNF processing represented on different layers.

\subsection{Network Graph Transformation}

We assume that $F C=f_{1}, f_{2} \ldots f_{k}$ denotes the network function chain that needs to be realized on the network topology, where $F C \subset F$. We transform the network graph into a "layered graph" by adding $k$ layers to the graph (counting the existing graph as the base layer), where $k=\operatorname{count}(F C)$ and each layer is an exact copy of the original graph. For every vertex $v$ in the original graph, let $v^{i}$ denote the corresponding node in the $i^{t h}$ layer $(i=0 \ldots k)$. Every $(i-1, i)$ layer pair is connected vertically only by edges between nodes $v^{i-1}$ and $v^{i}$ if that node provides the particular network function required by $f_{i}$. These vertical, directed edges are weighted by a cost $c(f, v)$ that is defined by the processing cost for utilizing network function $f$ on node $v$.

Figure 1 illustrates this transformation. In this example, we consider a service chain with two functions, i.e., $F=$ $f_{1}, f_{2}$. Function $f_{1}$ is available at node $B, H, I$ and $f_{2}$ is available only at node $F$. Consequently, we construct three layers of the original graph. The first layer is the base layer of the network topology. The second layer is connected to the first layer only through nodes that provide processing for $f_{1}$, in this case, nodes $B, H, I$. Finally, the last layer is connected to the second layer through node node $F$ that provides processing for $f_{2}$. These edges are weighted by their respective function processing costs.

\subsection{Adaptive Service Routing Algorithm}

It is always beneficial to reduce a problem to a certain state that can then be solved by an existing, optimized algorithm than formulating a completely new algorithm. In our approach described above, a conventional shortest path algorithm requires a slight modification to perform path-finding. The source node $s r c$ is placed in the first layer of the layered graph and the destination node $d s t$ is placed in the $k+1^{t h}$ layer. Shortest-path routing is then conducted across all layers from $s r c$ to $d s t$.

In the example in Figure 1, the goal is to find the shortest path from node $A$ to node $J$. After constructing the layered graph, we find a path from node $A_{s r c}^{0}$ in the first layer to node $J_{d s t}^{2}$ in the last layer. The structure of the layered graph forces traversal of vertical edges in order to reach the destination in the last layer. (Since edges are directed, no loops across layers can occur.) Here, nodes $B, H, I$ provide processing for $f_{1}$ and hence $\left(B^{0}, B^{1}\right),\left(H^{0}, H^{1}\right),\left(I^{0}, I^{1}\right)$ are the only edges that connected the first and second layers. The same argument applies to the second and third layers that are connected by $\left(F^{1}, F^{2}\right)$.

The cross-layer path that Dijkstra's returns can be mapped to a path in the original graph by folding nodes with layer qualifiers (the superscripts) back into their base nodes. Here, Dijkstra's algorithm returns $A \rightarrow B \rightarrow B^{1} \rightarrow E^{1} \rightarrow F^{1} \rightarrow$ $F^{2} \rightarrow J^{2}$, indicating that node $B$ was picked for processing $f_{1} \in F C$ and node $F$ was used for processing $f_{2} \in F C$. This path, nodes in lower layers overlaid on their originals, $A \rightarrow B \rightarrow E \rightarrow F \rightarrow J$, with the added processing costs at nodes $B, F$ for $f_{1}, f_{2}$ respectively.

The proof of correctness is inherent in the methodology itself. The minimum cost path from the $s r c$ node in the base layer to the $d s t$ node in the $k+1^{t h}$ is an overlay of an actual path in the original, untransformed network graph. The costs of both these paths are the same, since corresponding edges in all the layers have the same edge weights, which implies that there cannot be another least cost path. Furthermore, if there were some other least cost path, then it would have to translate into the same path over all the layers as discussed previously, which is in clear contradiction of the least cost path obtained by Dijkstra's.

\subsection{Partially Constrained Service Order- ing}

Normally when VNF chains are described, there is a certain order associated with them since data streams from the egress interface of one VNF will match up to the ingress interface of the succeeding VNF. Under some circumstances, when the interfaces are format agnostic, the ordering can be relaxed. We can simply solve this problem by constructing the layered graph as per the specified order and process it as described in Section 4.2. We can also treat the problem as one with a lower level of combinatorial complexity than the original, since we do not need to calculate all the possible combinations of VNFs and node sites, but just for the subsets of VNFs that do not care about order. Subsequently, we construct a layered graph as illustrated in Figure 2 for relaxed orders of $f_{2}, f_{3}$ and $f_{3}, f_{2}$ in a similar service chain as above. The VNFs are located at $f_{1}=A$, $f_{2}=B, f_{3}=D, E, f_{4}=E$. We interchange the ordering 


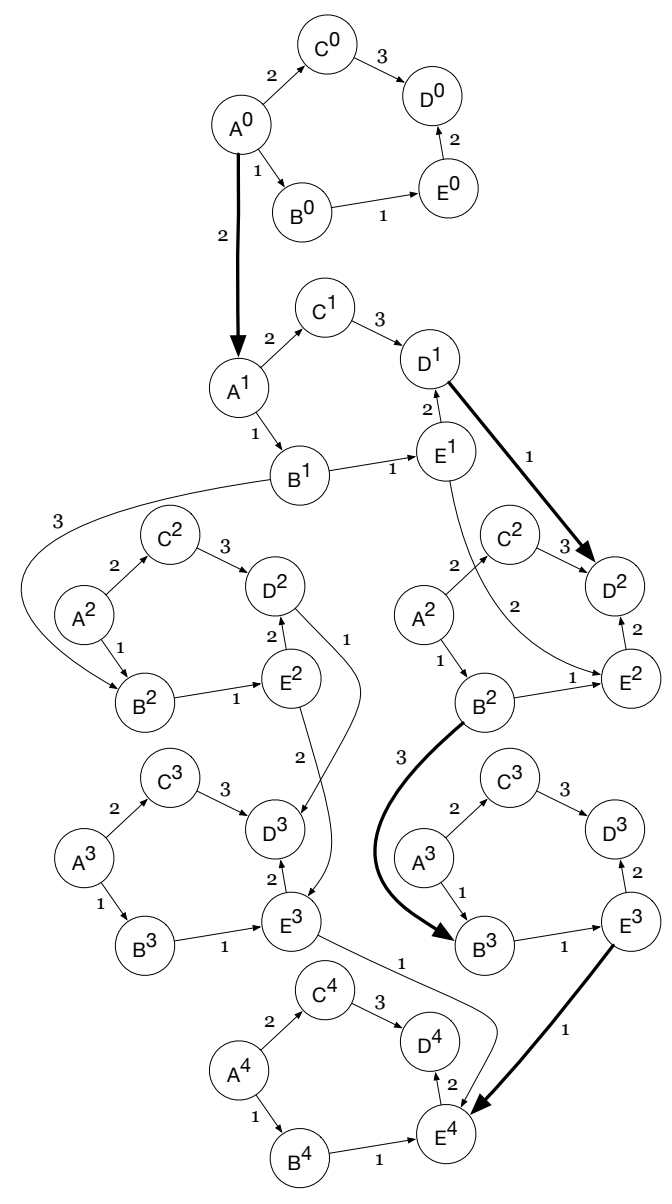

Figure 2: Relaxed VNF Ordering

in the middle two layers to accommodate both $f_{2}, f_{3}$ and $f_{3}, f_{2}$. The path-finding algorithm is free to choose a path as dictated by current network and nodal processing conditions.

The path-finding algorithm is then free to choose a path that is wants at layers that offer branching. For example, Dijkstra's can choose to go with either the $f_{2}, f_{3}$ on the left branch or the $f_{3}, f_{2}$ on the right branch Figure 2. In this approach, the question of space complexity does arise, since we are principally storing multiple copies of the graph as layers, not just with our vertical layering, but with the horizontal layering being introduced here. Graph storage optimizations can be used to remedy this, but those discussions are beyond the scope of this paper.

\subsection{Delay Modeling}

In our Adaptive Service Routing algorithm, we use delay as the cost metric, consisting of the sum of communication delays on links plus processing delays on VNF nodes.

Note that the use of delay as cost metric can lead to instability in some network routing protocols. This, however, is not a concern here since traffic does not get rerouted because of changes in load. Only new connections consider current delays and load on processing nodes. Once placed, a connection stays on the same path till it expires or is removed. If a flow traverses a link multiple times (e.g., on different layers due to a specific selection of processing nodes), the added queuing delay cost of the multiple instances of this flow are not considered.

\subsubsection{Communication Delay}

The communication delay for traversing a single link consists of the following three components: (1) queuing delay $d_{\text {queue }}$, (2) transmission delay $d_{t x}$, and (3) propagation delay $d_{\text {prop }}$. The transmission delay is

$$
d_{t x}=p k t s i z e / b w,
$$

where pktsize is the size of the transmitted packet and $b w$ is the link bandwidth. The propagation delay is

$$
d_{\text {prop }}=l / c_{\text {medium }},
$$

where $l$ is the physical length of a link and $c_{\text {medium }}$ is the propagation speed of signals in that medium.

The queuing delay at a node is dependent on the egress interface load, denoted by loadlink. The load is the ratio of allocated bandwidth (from previous connections) to the total link bandwidth. Using a simple M/M/1 queuing model with an expected service time of $d_{t x}$, we get a queuing delay of

$$
d_{\text {queue }}=\operatorname{load}_{\text {link }} /\left(1-\operatorname{load}_{\text {link }}\right) * d_{t x} .
$$

We use a superscript to denote the delay values for a specific node and edge. The total cost associated with an edge is thus $c\left(e_{i}, e_{j}\right)=d_{q u e u e}^{e i}+d_{t x}^{e i, e j}+d_{t x}^{e i, e j}$.

\subsubsection{Processing Delay}

The nodal processing delay is time taken to process a packet on the network node. The advent of powerful network processors with their multi-processing capabilities etc. has managed to keep the processing delay under a manageable threshold on traditional network nodes, despite the increased time it takes for complex packet processing functions.

We can model processing delay based on Generalized Processor Sharing (GPS):

$$
d_{\text {proc }}=\left(\text { load }_{\text {processor }} / \text { load }_{\text {flow }}\right) * t_{\text {proc }}
$$

where load $d_{\text {processor }}$ is the current load on the processor expressed in percent, load $_{\text {flow }}$ is an approximate fraction of the load the flow will contribute to the processor, and $t_{\text {proc }}$ is the per-packet processing time (e.g., from experimentation or equations in [11]).

In practice, balancing load and avoiding allocations that utilize systems at their maximum capacity may be desirable.

$$
d_{\text {proc }}=\operatorname{load}_{\text {proc }} /\left(1-\text { load }_{\text {proc }}\right) * t_{\text {proc }} .
$$

An M/M/1 queuing model captures the nearly linear growth in delay for low loads and associated high costs near system capacity. We use this for our hypothesis. Other queuing models may be used as necessary.

\section{EVALUATION}

In our evaluation, we first present a simple scenario to show the validity of our approach. We then discuss our implementation on an emulated framework. 


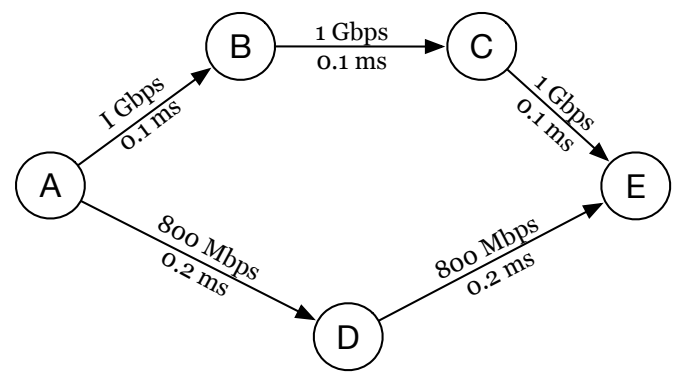

Figure 3: Network topology for example scenario.

\subsection{Theoretical Model}

To demonstrate the effectiveness of our proposed approach, we show its operation on a small network topology. In this example, the various effects of resource load, delay modeling, and node and path choices are illustrated.

The network topology in Figure 3 shows the performance characteristics of the links and processing nodes. We assume that connections need to be routed from $A$ to $E$ and a VNF needs to be used (available on nodes $B, C$, and $D$ ).

Figure 4 shows results of repeatedly mapping a $30 \mathrm{Mbps}$ service request between nodes $A$ and $E$ onto the topology. The first 23 connection requests are assigned to the upper path $(A-B-C-E)$ due to the lower latency on that path. Note that processing allocation is split between nodes $B$ and $C$ proportional to their capacity. As the load increases on the upper path, the lower path $(A-C-E)$ is preferred and requests 24 through 56 are allocated to both paths (with the majority going to the lower path). As the network reaches its capacity, the final requests are allocated to the top path.

Figure 5 compares our algorithm with a number of other routing techniques. All approaches accommodate fewer connections (random: 43, round robin: 48, static $B: 13$, static $C: 19$, static $D: 26$ ) than our Adaptive Service Routing algorithm with 58. Also, our algorithm consistently achieves lower delays across all mappings (random and round robin achieve some lower delays in later mappings because earlier ones use resources on clearly suboptimal paths.)

We also measure the processing time required by the algorithm to make path-finding decisions. The topologies chosen here are Waxman, Barabasi-Albert and GLP models of different sizes, generated using the BRITE topology generator [10]. Processing has been simulated for 3, 5 and 7 services in the VNF chain. Table 1 summarizes the results. We observe computational times in the order of half a minute for graph sizes of $10 \mathrm{~K}$ nodes and 7 VNFs chained together. It would be beneficial to note that these are large sized graphs with multiple VNF site deployments. Most of the computational time is taken up by layering (which is currently nonparallelized). We believe they can be lowered further using graph optimization and parallelization techniques.

\subsection{Prototype Implementation}

We have implemented this service routing technique on a practical, emulated software-defined network running in Mininet [8] and controlled by ONOS [1]. Linux's TCLink
Table 1: Adaptive Routing Path-Finding Times

\begin{tabular}{|l|r|r|r|}
\hline & \multicolumn{3}{|c|}{ Processing Time $(s)$} \\
\hline Topologies & 3 VNFs & 5 VNFs & 7 VNFs \\
\hline \hline Waxman-100 & 0.02 & 0.05 & 0.09 \\
Waxman-1K & 0.23 & 0.59 & 1.09 \\
Waxman-10K & 3.24 & 8.25 & 15.30 \\
\hline BA2-100 & 0.04 & 0.09 & 0.17 \\
BA2-1K & 0.46 & 1.16 & 2.12 \\
BA2-10K & 6.69 & 16.97 & 30.97 \\
\hline GLP-100 & 0.03 & 0.08 & 0.14 \\
GLP-1K & 0.41 & 1.02 & 1.85 \\
GLP-10K & 5.42 & 13.99 & 25.82 \\
\hline
\end{tabular}

library was used to define and constrain bandwidth and delay on the links. Due to Open vSwitch and Mininet limits on our hardware, emulated testing could be performed only for topologies with 60-70 switches and 20 hosts. Processing times for large topologies have been evaluated only for the algorithmic section as described in Section 5.1. We contend that the crux of the decision making is the graph transformation and the emulated testbed is just a method of instrumentation. We did observe that the practical scenarios do indeed follow our theoretical results for smaller topologies.

We use sFlow-RT and host-sFlow, instrumented flow sampling mechanisms of the sFlow standard [12], to obtain utilization of links and nodes. Figure 6 shows that our model from Section 4.4 matches with dynamic network data.

\section{SUMMARY AND CONCLUSION}

In this paper, we presented an Adaptive Service Routing algorithm that routes traffic adaptively through various VNF nodes in an SDN based on instantaneous network latency. We use an example to show how this adaptive routing can help balance loads on VNF processing throughout the network. Due to the use of a single metric and translating the routing problem into a simple shortest path problem on a larger graph, our algorithm can prove to be useful in largescale networks with multi-site VNF deployments.

\section{Acknowledgments}

This material is based upon work supported by the National Science Foundation under Grant No. 1421448.

\section{REFERENCES}

[1] Berde, P., Gerola, M., Hart, J., Higuchi, Y., Kobayashi, M., KoIde, T., LANTz, B., O’Connor, B., Radoslavov, P., SNOW, W., AND PARUlKar, G. M. ONOS: towards an open, distributed SDN OS. HotSDN (2014), 1-6.

[2] Casado, M., Freedman, M. J., Pettit, J., Luo, J., MCKEOWn, N., AND SHENKER, S. Ethane: taking control of the enterprise. In SIGCOMM '07: Proceedings of the 2007 conference on Applications, technologies, architectures, and protocols for computer communications (Kyoto, Japan, Aug. 2007), pp. 1-12.

[3] Chen, X., CAI, H., And Wolf, T. Multi-criteria routing in networks with path choices. In Proc. of 23rd IEEE International Conference on Network Protocols (ICNP) (San Francisco, CA, Nov. 2015). 


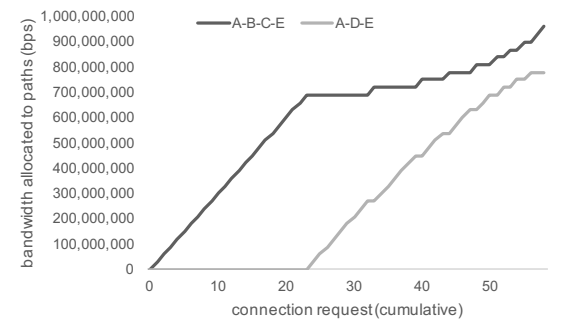

(a) Path allocation.

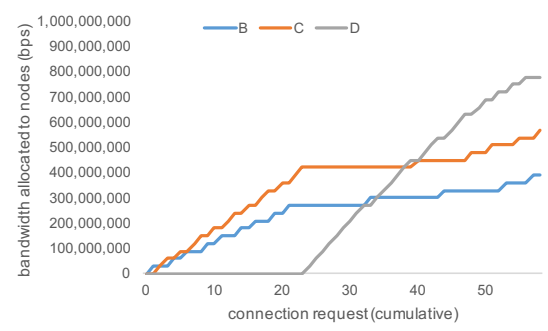

(d) Node allocation.

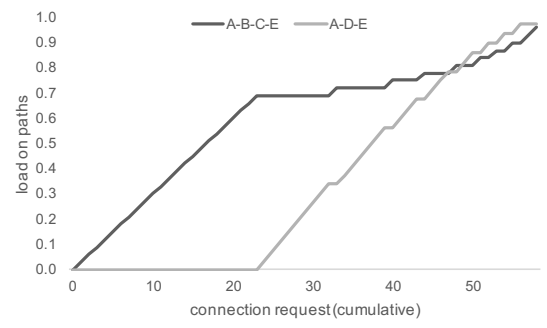

(b) Path load.

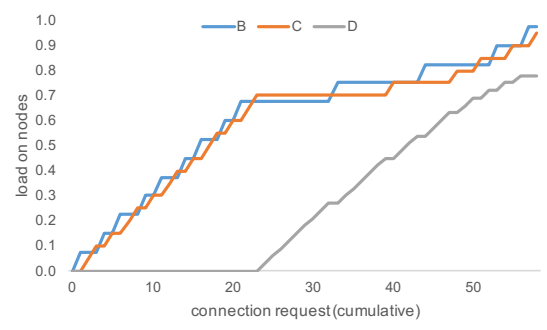

(e) Node load.

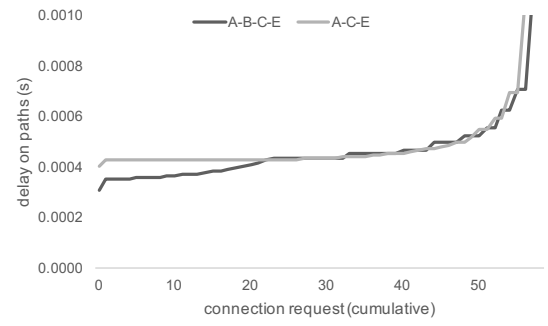

(c) Path delay.

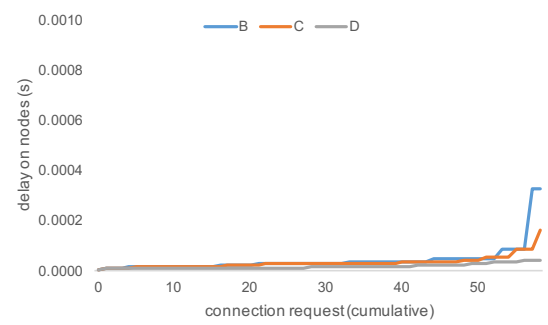

(f) Node delay.

Figure 4: Performance characteristics of repeated service routing on example topology.

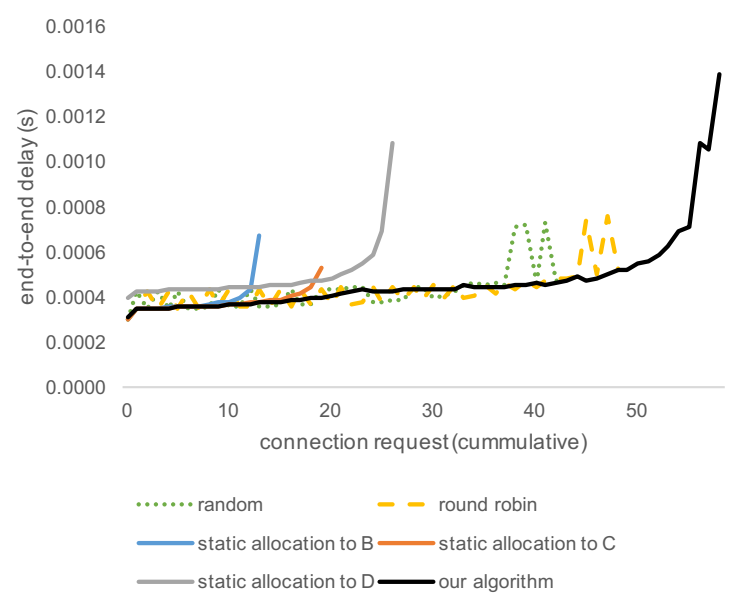

Figure 5: Comparison of VNF routing algorithms.

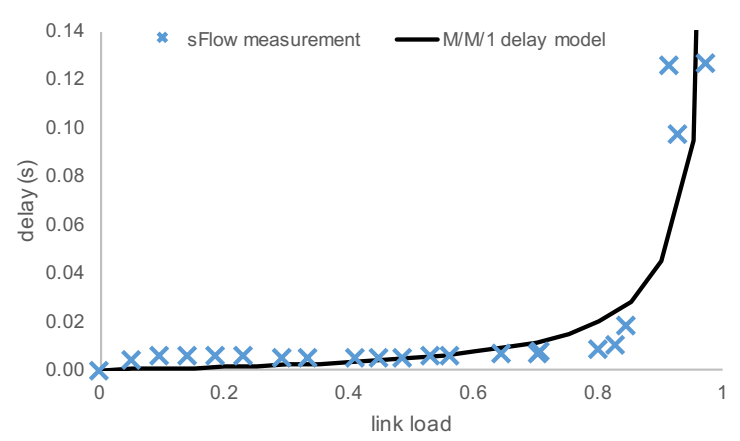

Figure 6: Delay model validation against sFlow data
[4] Choi, S. Y., Turner, J. S., AND Wolf, T. Configuring sessions in programmable networks. Computer Networks 41, 2 (Feb. 2003), 269-284.

[5] Dijkstra, E. W. A note on two problems in connexion with graphs. Numerische Mathematik 1 (Dec. 1959), 269-271.

[6] Dwaraki, A., AND Wolf, T. Service instantiation in an Internet with choices. In Proc. of the 22nd IEEE International Conference on Computer Communications and Networks (ICCCN) (Nassau, Bahamas, Aug. 2013).

[7] Ghaznavi, M., Khan, A., Shahriar, N., Alsubhi, K., AhMED, R., AND BouTABA, R. Elastic virtual network function placement. In Proc. of IEEE International Conference on Cloud Networking (CloudNet) (Niagara Falls, Canada, Oct. 2015), pp. 255-260.

[8] Lantz, B., Heller, B., AND McKeown, N. A network in a laptop: rapid prototyping for software-defined networks. HotNets (2010), 19-6.

[9] McKeown, N., Anderson, T., Balakrishnan, H., Parulkar, G., Peterson, L., Rexford, J., Shenker, S., AND TURNER, J. OpenFlow: enabling innovation in campus networks. SIGCOMM Computer Communication Review 38, 2 (Apr. 2008), 69-74.

[10] Medina, A., Lakhina, A., Matta, I., Byers, J. M. A., OF COMPUTER, S., AND ON, T. S. . P. N. I. S. BRITE: an approach to universal topology generation. In Modeling, Analysis and Simulation of Computer and Telecommunication Systems, 2001. Proceedings. Ninth International Symposium on (2001), IEEE Comput. Soc, pp. 346-353.

[11] Ramaswamy, R., Weng, N., and Wolf, T. Characterizing network processing delay. In Proc. of IEEE Global Communications Conference (GLOBECOM) (Dallas, TX, Nov. 2004), pp. 1629-1634.

[12] sFlow Standard v5. http://sflow.org/sflow_version_5.txt.

[13] WOLF, T. Service-centric end-to-end abstractions in next-generation networks. In Proc. of Fifteenth IEEE International Conference on Computer Communications and Networks (ICCCN) (Arlington, VA, Oct. 2006), pp. 79-86. 